REVISTA DE ESTUDIOS E INVESTIGACIÓN EN PSICOLOGÍA Y EDUCACIÓN

\title{
Modelos mentales en alumnado con TDAH: Competencias lectora y matemática
}

\section{Mental models in students with ADHD: Reading and mathematical skills}

Dolores Valerazo (D) https://orcid.org/0000-0002-5903-1560

Pilar Vieiro (iD) https://orcid.org/0000-0003-4450-7308

Facultad de Ciencias de la Educación, Universidad de A Coruña

https://www.educacion.udc.es/

A Coruña - España 


\title{
Resumen
}

Los niños con trastorno por déficit de atención e hiperactividad (TDAH) presentan una alta comorbilidad con dificultades de aprendizaje de la lectura y de las matemáticas, y una baja capacidad en relación con la planificación, organización e inhibición de la respuesta, y el mantenimiento de la concentración en la tarea. En este contexto, el objetivo de este estudio transversal es analizar la influencia de los modelos mentales en tareas de resolución de problemas matemáticos, cálculo y procesamiento semántico. La muestra estaba integrada por un total de 304 alumnos de Educación Primaria, 152 de tercer curso y 152 de sexto; la mitad formaban el grupo TDAH y la otra mitad el grupo control. Como instrumento de medida del procesamiento semántico se utilizó el programa Gesmedición, y para evaluar la competencia matemática la prueba de Problemas Verbales Aritméticos (PVA). Los resultados mostraron diferencias significativas en eficacia a favor del grupo control. Los sujetos con TDAH de ambos niveles educativos se mostraron significativamente más eficaces en tareas de cálculo, siendo los niveles de eficacia más bajos los de resolución de problemas, invirtiendo el patrón en relación al control.

Palabras clave: TDAH; cálculo matemático; resolución de problemas matemáticos; semántica y modelos mentales

\begin{abstract}
Children with attention deficit hyperactivity disorder (ADHD) display high comorbidity with learning difficulties in reading and mathematics and low ability in relation to response planning, organisation and inhibition, and maintaining task focus. In this context, the aim of this cross-sectional study is to analyse the influence of mental models on mathematical problem-solving, calculation and semantic processing tasks. The sample comprised a total of 304 primary school pupils, 152 from 3rd class and 152 from 6th class; half made up the ADHD group and the other half the control group. Semantic processing was measured using the Gesmedición programme and mathematical competence was measured using the Verbal Arithmetic Problems test. The results showed significant better efficacy in among the control group. The pupils with ADHD at both educational levels were significantly more effective in calculation tasks, with the lowest levels of effectiveness being those of problem solving, reversing the pattern in relation to the control group.
\end{abstract}

Keywords: ADHD; mathematical calculation; mathematical problem-solving; semantics and mental models 
Los niños con TDAH suelen experimentar problemas académicos bien por la falta de atención, bien por la hiperactividad (Bauermeister et al., 2005; Frazier et al., 2007; MirandaCasas et al., 2006). Los estudios que han investigado las comorbilidades de TDAH y dificultades de aprendizaje, sugieren que entre el 31 al $45 \%$ de los estudiantes con TDAH tienen dificultades de aprendizaje o viceversa; de entre dichas dificultades caben destacar las relacionadas con el aprendizaje del lenguaje escrito y de las matemáticas (Compto, 2012; DuPaul et al., 2013). En este sentido, la literatura científica muestra que los niños con TDAH tienen una comprensión de lectura más pobre que sus compañeros debido a un déficit en la asignación estratégica de la atención, de la planificación y en el procesamiento de información (Alloway, 2004; Pennington y Ozonoff, 1996). Dichos problemas parecen ser independientes de cualquier dificultad con la lectura de palabras.

El vínculo entre el déficit de atención y las dificultades matemáticas ha sido investigado en diversos trabajos (Kaufmann y Nuerk, 2008; Passolunghi et al., 2005; Rubinsten y Henik, 2009, entre otros). En algunos se han encontrado asociaciones entre los síntomas del TDAH y los trastornos del aprendizaje en estudios de cohortes y de gemelos con lo que se demostró un riesgo genético compartido entre el TDAH y capacidad matemática reducida (Czamara et al., 2013; Greven et al., 2014). Sin embargo, la hipótesis causal mayormente defendida a cerca de la etiología y la relación entre las dificultades en el aprendizaje instrumental de la lectura y las matemáticas proviene de la explicación de que existe una alteración del bucle fonológico al procesar información de carácter verbal en alumnos con dificultades en el aprendizaje de las matemáticas, pudiendo alterar la coordinación de muchas tareas numéricas y la resolución de problemas (Alderson et al., 200). En este contexto Cragg y Gilmore (2014) destacan la importancia de ciertas habilidades tales como las funciones ejecutivas (la amplitud de memoria de trabajo, la capacidad para eliminar información irrelevante que pueda distraer_inhibir_y la capacidad de modificar la estrategia si el caso lo requiere) en la adquisición y evolución de la competencia matemática (Cowan et al., 2011; Cragg et al., 2014; Geary et al., 2004; Orbach et al,. 2020; Polanczyky, 2014). En el caso de la competencia matemática el proceso de planificar se muestra fundamental para mantener la información crítica disponible y organizar la manera de llegar a la solución para posteriormente introducir los procesos de cálculos correspondientes (Geary, 2011; Dayson et al., 2013; Marzocchi et al., 2002). La hipótesis más aceptada acerca de esta relación causal parece estar vinculada con un mal ajuste en el mecanismo inhibitorio que posibilita el suprimir la información irrelevante del sistema, así como con las dificultades que presentan los estudiantes con TDAH para concentrarse en los estímulos significativos o 
enmascarados (Passolunghi y Siegel, 2004; Marzocchi et al., 2002). En este sentido los errores que se cometen en tareas de resolución de problemas, podría deberse a su incapacidad de inhibir información irrelevante que estaría ocupando un espacio importante en la memoria de trabajo. De este modo se vería limitada la habilidad para tomar decisiones en tareas de resolución de problemas matemáticos. Ciertos investigadores mantienen que incluso en tareas donde la información textual está ausente, véase el cálculo, la capacidad de generar modelos mentales predice el rendimiento matemático (Bull et al., 2008). No obstante, se producen cambios evolutivos en el desarrollo de la función cognitiva, visibles también en las funciones ejecutivas de los sujetos con TDAH y niños con dificultades de aprendizaje. De este modo se encontraron cambios sustanciales en la memoria de trabajo relacionadas con el cálculo matemático (Brocki y Bohlin, 2004; Friedman et alt., 2018) demostrando una interacción entre la inhibición y la memoria de trabajo en el desarrollo del funcionamiento ejecutivo en este tipo de tareas.

En este contexto el objetivo, general de este trabajo es analizar la influencia de la activación de modelos mentales en la eficacia de alumnado con TDAH en situaciones experimentales que demanden activación de modelos mentales, véase resolución de un problema matemático vs identificación de inferencias, agregando una condición experimental ajena al lenguaje escrito, pero sí a la representación numérica y por ende a la generación de un modelo mental como es el cálculo. Y de este modo comprobar si se produce ese cambio evolutivo indicado en diversas investigaciones acerca de la influencia diferencial de los modelos mentales en las operaciones aritméticas en población con TDAH.

\section{Método}

\section{Participantes}

La muestra estuvo conformada por un total de 304 participantes, 152 de los cuales estaban escolarizados en 3ㅇ de Educación Primaria (EP) y los 152 restantes en 60 de EP. Todos ellos procedían de colegios públicos y concertados, situados en la comunidad de Galicia y estaban escolarizados en los mismos desde primer curso de Educación Infantil. En cada nivel educativo 76 estaban diagnosticados de TDAH y otros 76 actuaron como grupo control. La media de edad de los sujetos con TDAH fue de $8.5(D T=1.02)$ y $11.52(D T=1.22)$ respetivamente y de los sujetos control de $8.82(D T=1.59)$ y $11.34(D T=1.74)$.

Los estudiantes con TDAH fueron seleccionados atendiendo a su diagnóstico clínico profesional, ya fuera psiquiátrico o neuropsiquiátrico, en la mayoría de los casos, o psicológico. 


\section{Instrumentos}

Gesmedición (Vieiro et al., 2011): prueba on-line que tiene como propósito evaluar los procesos de lectoescritura (léxico, sintáctico y semántico) y registra la tasa de tiempos de reacción y aciertos, y por ende eficacia y eficiencia en dichas tareas. Para el presente estudio sólo utilizaremos los resultados de las tareas de semántica. La prueba evalúa la capacidad de reconocimiento textual y la generación de inferencias (la activación de un modelo mental).

Problemas Verbales Aritméticos (PVA) de Artiles y Jiménez (2011): el propósito de esta prueba es conocer el nivel de competencia de los niños de Educación Primaria en resolución de problemas verbales aritméticos. Está compuesta por 33 problemas, de los cuales 20 se clasifican en cuatro categorías de problema (combinación, comparación, igualación y cambio) y los 13 problemas restantes se clasifican en cuatro categorías (comparación, conversión, combinación y problemas de razón). Los problemas se resuelven utilizando una sola operación aritmética (suma, resta, multiplicación o división).

\section{Diseño}

El diseño incluyó la comparación de grupos a fin de analizar si existían diferencias a la hora poner en marcha estrategias de comprensión relacionadas con la generación de un modelo mental en tareas cálculo, resolución de problemas matemáticos y comprensión de oraciones. Asimismo, dichas medidas fueron analizadas en un estudio de corte transversal.

\section{Procedimiento}

Para la identificación del grupo control se solicitó una entrevista con los directores de los CEIP seleccionados, con el fin de explicar las características de la investigación y obtener la colaboración del personal docente. Posteriormente, se obtuvo la autorización por escrito de los padres antes de dar comienzo al estudio.

La selección del grupo experimental se realizó a través de una asociación de TDAH, quien nos proporcionó datos de contacto con las familias quienes del mismo modo que en el grupo control dieron su consentimiento informado para la participación de sus hijos/as en el estudio.

Una vez reclutadas ambas muestras se administraron de modo global la tarea semántica del Gesmedición y la prueba Problemas Verbales Aritméticos (Artiles y Jiménez, 2011).

Se aclaró a los niños que su participación era voluntaria y anónima. No se presentaron negativas. 


\section{Resultados}

Los datos obtenidos fueron analizados con el IBM SPSS, v. 26.0. En primer lugar, se procedió a un análisis descriptivo de los resultados de puntuaciones obtenidos en promedios, máximas, mínimos y desviación en aciertos en los distintos procesos (cálculo, resolución de problema y semántica) en los grupos de control y TDAH (experimental), como muestra en las Tablas 1 y 2.

Como era de esperar, el grupo control ha sido el más eficaz en todas las tareas tanto de semántica como de cálculo y resolución de problemas matemáticos. El patrón de eficacia en 3으 de EP (Tabla 1) ha sido el siguiente: el grupo control ha obtenido unas tasas más altas de aciertos en semántica y menos en resolución de problemas y cálculo por este orden, frente al grupo con TDAH donde los resultados se han invertido, los niveles más altos se situaron en cálculo (por encima de la media) y los menores en semántica y cálculo (claramente por debajo de la media).

Tabla 1

Análisis descriptivo del grupo control y TDAH en 3o de Educación Primaria

\begin{tabular}{lcccccccc}
\hline & \multicolumn{4}{c}{ Control } & \multicolumn{4}{c}{ TDAH } \\
\cline { 2 - 8 } & $M$ & Máx & Mín & $D T$ & $M$ & Máx & Mín & $D T$ \\
\cline { 2 - 8 } Cálculo & 80.34 & 100.00 & 52.00 & 7.53 & 51.60 & 90.00 & 33.00 & 8.28 \\
Problemas & 82.19 & 100.00 & 53.00 & 9.61 & 31.12 & 78.00 & 31.00 & 9.42 \\
Semántica & 95.79 & 100.00 & 73.00 & 8.93 & 38.46 & 52.17 & 21.35 & 8.60 \\
\hline
\end{tabular}

En contraste de medias a través de la prueba $t$-Student mostró que en el grupo de 30 de EP las diferencias entre el grupo TDAH y el control fueron estadísticamente significativas en todas las medidas a favor de este último (Cálculo: $t=4.61, p<.001$; Resolución de problemas: $t$ $=3.04, p<.001$; tareas semánticas: $t=4,26, p<.001$; unilateral). Igualmente sucedió en el grupo de 60 de EP (Tabla 2) (Cálculo: $t=4.61, p<.009$; Resolución de problemas: $t=1.047, p<.03$; tareas semánticas: $t=3.66, p<.01$; unilateral).

\section{Tabla 2}

Análisis descriptivo del grupo control y TDAH en 6 de Educación Primaria

\begin{tabular}{lcccccccc}
\hline & \multicolumn{4}{c}{ Control } & \multicolumn{4}{c}{ TDAH } \\
\cline { 2 - 8 } & $M$ & Máx & Mín & $D T$ & $M$ & Máx & Mín & $D T$ \\
\cline { 2 - 8 } Cálculo & 81.84 & 100.00 & 70.00 & 3.63 & 60.66 & 100.00 & 30.00 & 8.38 \\
Problemas & 81.05 & 100.00 & 75.00 & 6.01 & 34.08 & 90.00 & 28.00 & 5.46 \\
Semántica & 97.74 & 100.00 & 85.00 & 1.97 & 41.46 & 79.17 & 33.33 & 8.65 \\
\hline
\end{tabular}

El patrón de resultados es diferente dentro de cada uno de los grupos (TDAH vs Control) aunque se mantiene en ambos niveles educativos. Las tareas semánticas fueron 
significativamente más fáciles para los sujetos Control que las tareas de resolución de problemas o de cálculo tanto en 3ำ de EP (Anova: $F=13.0 ; p<.001$; Pruebas de Scheffé, $p<.01$, en todos los casos) como en 60 de ESO (Anova: $F=17.32 ; p<.001$; Pruebas de Scheffé, $p<.01$, en todos los casos). Por su parte las tareas de cálculo fueron significativamente más fáciles para los sujetos TDAH que las tareas de resolución de problemas y semánticas tanto en 3ำ de EP (Anova: $F=11.8 ; p<.001 ;$ Pruebas de Scheffé, $p<.001$, en todos los casos) como en 6 o de ESO (Anova: $F=14.44 ; p<.001 ;$ Pruebas de Scheffé, $p<.001$, en todos los casos).

Evolutivamente se observa un incremento en la eficacia en ambos grupos (experimental vs. control) en el nivel de 6 ㅇ de Educación Primaria con relación al de 3o pero en ningún caso las diferencias lograron el nivel de significación estadística.

\section{Discusión}

Tal y como hemos analizado al inicio de este trabajo la adquisición de la competencia matemática requiere atención sostenida para memorizar hechos y secuencias de pasos mientras se autocontrola y revisa las respuestas, al mismo tiempo requiere en tareas de resolución de problemas no sólo una respuesta a una situación matemática (comprensión de la estructura, aplicación de la operación, interpretación de un esquema cuantitativo, que nos lleva aplicar procedimientos) sino que también necesitamos de un esquema mental eficiente y coherente para resolver la situación problemática que se nos plantea.

Algunos estudios han venido demostrando que dicho modelo mental condiciona igualmente, aunque de distinto modo, la eficacia en la resolución del cálculo aritmético (Brocki y Bohlin, 2004; Friedman et al., 2018). En este sentido, nuestro estudio no corrobora los citados estudios pues los alumnos con TDAH tienen un rendimiento mayor en calculo, situándose éste por encima de la media aun siendo menos eficaces que los controles.

Las tareas de cálculo resultaron ser las más fáciles para los sujetos con TDAH y las de resolución de problemas más difíciles. Interpretamos estos datos en el marco de las demandas de la tarea: la resolución de problemas y la generación de inferencias están condicionadas por la generación de un modelo mental que pueda ayudar considerar cognitivamente manejable una situación más compleja.

Las tareas de resolución de problemas implican una doble o incluso triple sobrecarga cognitiva: son tareas mediatizadas por la lectura, implican la generación de un modelo mental para la comprensión del enunciado que obliga a decidir al sujeto qué tipo de operación matemática debe aplicar que, finalmente, ha de ser resuelta. 
Se observa, por tanto, que en los sujetos con TDAH la posible sobrecarga cognitiva de la tarea resultante del proceso de lectura y de la combinación aritmética, dificulta su nivel de eficacia, no viéndose esta tarea beneficiada de modo significativo por la edad.

En el grupo control las tareas más difíciles son igualmente las de resolución de problemas, pero, a diferencia de los TDAH las tareas semánticas se presentan como las más fáciles, parece por tanto que en este grupo no es el modelo mental el que mediatiza más fuertemente la eficacia en la resolución de problemas matemáticos.

Para explicar por qué el TDAH puede estar asociado negativamente con aprender (memoria y comprensión) podemos basarnos en los trabajos de Fernández (2019), Kofler et al. (2013) o Molinar y Cervantes (2021). Según estos autores si un niño no toma nota de lo que está sucediendo y no escucha, no interiorizará la información como otros. La atención y el autocontrol, carentes o deficitarios en TDAH, son entendidos como atributos académicos positivos y, en consonancia, pueden considerarse como un indicador de compromiso cognitivo, es decir como la clave para aprender (Mayer, 2004).

A la luz de estos resultados podríamos estar en disposición de afirmar que las demandas cognitivas asociadas a una tarea de lectura que influyen de modo significativo en toda la población lo hacen de modo especial en la población con TDAH. Ello limita las habilidades de comprensión necesarias para seleccionar información y generar un modelo mental adecuado a las exigencias de la tarea. Se vería, por lo tanto, condicionada su capacidad para manejar múltiples operaciones y transformar la información verbal dentro de los problemas. A ello se puede sumar, tal y como indican los estudios de Kaufmann et al., (2008) o Tymms \& Merrell (2011) el cálculo lento característico de los TDAH con el consiguiente aumento en la carga atencional.

Además, nuestros datos demuestran es que loa sujetos con TDAH independientemente del nivel educativo presentan un bajo rendimiento en tareas que requieren de la activación de un modelo mental más complejo, no coincidiendo con los trabajos de Brocki y Bohlin (2004) o de Friedman et al. (2018), lo cual se puede atribuir tanto al ciclo evolutivo seleccionado como a que la diferencia de edad entre ambos grupos era únicamente de 3 años, con relación a lo primero considerábamos al grupo de 60 de EP como perteneciente a una etapa donde el desarrollo metacognitivo comenzaba a desarrollarse. Una investigación con más control de variables de esta naturaleza en población TDAH nos podría arrojar más datos al respeto.

En cuanto a la capacidad operacional aritmética esta no se ve afectada, a pesar de que sin duda alguna también requiere un cierto modelo mental pero donde se pueden aplicar más fácilmente rutinas aprendidas previamente. En este sentido nuestros resultados contradicen los 
encontrados por Bull et al., (2008) acerca de que incluso en tareas donde la información textual está ausente la capacidad de generar modelos mentales predice el rendimiento en TDAH.

\section{Conclusiones}

Los alumnos con TDAH presentaron un rendimiento inferior a sus compañeros normotípicos en tareas de aprendizaje de las matemáticas y comprensión lectora manifestando un patrón evolutivo constante en alumnado de segundo y tercer ciclo de Educación Primaria.

Las tareas donde se manifiestan más eficaces son las de cálculo, superando la media en aciertos; por el contrario, los peores resultados se encontraron en resolución de problemas. En este sentido, se confirma nuestra hipótesis acerca de la importancia del modelo mental en resolución de tareas semánticas (generación de inferencias) y problemas matemáticos que contribuyen a la percepción y organización de la realidad, así como a las construcciones simbólicas. Es decir, estructuran el conocimiento previo en los procesos de comprensión tan necesarios para comprender un problema e integrar un texto.

A su vez los análisis sugieren que existe alguna ventaja para niños más mayores en la resolución de las tareas propuestas, aunque nunca llegando a alcanzar los niveles de eficacia de los controles.

En este último sentido sería interesante prolongar el estudio transversal con muestras de alumnado de Educación Secundaria y comprobar si el patrón de resultados se mantiene.

\section{Referencias}

ALDERSON, R. Matt, RAPPORT, Mark. D, \& KOFLER, Michael. J. (2007). Attentiondeficit/hyperactivity disorder and behavioral inhibition: a meta-analytic review of the stop-signal paradigm. Journal of Abnormal Child Psychology, 35(5), 745-758. https://doi.org/10.1007/s10802-007-9131-6

ALLOWAY, Tracy P.; GATHERCOLE, Susan E.; WILLIS, Catherine; \& ADAMS, Anne M. (2004). A structural analysis of working memory and related cognitive skills in young children. Journal of Experimental Child Psychology, 87(2), 85-106. https://doi.org/10.1016/j.jecp.2003.10.002

ARTILES, Ceferino, JIMÉNEZ, Juan E. (2011). PVA: Problemas Verbales Aritméticos. En: Ceferino Artiles y Juan E. Jiménez, Normativización de instrumentos para la detección e identificación de las necesidades educativas del alumnado con trastorno por déficit de atención con o sin hiperactividad (TDAH) o alumnado con dificultades específicas de aprendizaje (DEA) (pp. 2744). Consejería de Educación, Universidades y Sostenibilidad. Dirección General de Ordenación, Innovación y Promoción Educativa. Disponible en: https://www.gobiernode 
canarias.org/educacion/web/servicios/necesidades_apoyo_educativo/otras_neae/publica ciones/publicacion_00574/

BAUERMEISTER, José J.; MATOS, Maribel, REINA, Graciela, SALAS, Carmen. C., MARTíNEZ, José V., CUMBA, Eduardo, \& BARKLEY, Russell A. (2005). Comparison of the DSM-IV combined and inattentive types of ADHD in a school-based sample of Latino/Hispanic children. Journal of Child Psychology and Psychiatry, 46(2), 166-179. https://doi.org/10.1111/ j.1469-7610.2004.00343.x

BROCKI, Karin C., \& BOHLIN, Gunilla (2004). Executive functions in children aged 6 to 13: A dimensional and developmental study. Developmental Neuropsychology, 26(2), 571-593. https://doi.org/10.1207/s15326942dn2602_3

BULL, Rebecca; ESPY, Kimberly; \& WIEBE, Sandra (2008). Short-term memory, working memory, and executive functioning in preschoolers: Longitudinal predictors of mathematical achievement at age 7 years. Developmental Neuropsychology, 33(3), 205-228. https://doi. org/10.1080/87565640801982312

COMPTON, Donald L., FUCHS, Lynn S., FUCHS, Douglas, LAMBERT, Warren, \& HAMLETT, Carol (2012). The cognitive and academic profiles of reading and mathematics learning disabilities. Journal of Learning Disabilities, 45(1), 79-95. https://doi.org/10.1177/0022219410393012

COWAN, Nelson (2011). The focus of attention as observed in visual working memory tasks: Making sense of competing claims. Neuropsychologia, 49(6), 1401-1406. https://doi.org/ 10.1016/j.neuropsychologia.2011.01.035

CRAGG, Lucy, \& GILMORE, Camilla (2014). Skills underlying mathematics: The role of executive function in the development of mathematics proficiency. Trends in Neuroscience and Education, 3(2), 63-68. https://doi.org/10.1016/j.tine.2013.12.001

CZAMARA, Darina; TIESLER, Carla; KOHLBÖCK, Gabriele; BERDEL, Dietrich; HOFFMANN, Barbara; BAUER, Carl; \& HEINRICH, Joachim (2013). Children with ADHD symptoms have a higher risk for reading, spelling and math difficulties in the GINIplus and LISAplus cohort studies. PloS one, 8(5), 63859. https://doi.org/10.1371/journal.pone.0063859

DUPAUL, George. J., GORMLEY, Matthew. J., \& LARACY, Seth. D. (2013). Comorbidity of LD and ADHD: Implications of DSM-5 for assessment and treatment. Journal of Learning Disabilities, 46(1), 43-51. https://doi.org/10.1177/0022219412464351

DYSON, Nancy I., JORDAN, Nancy C., \& GLUTTING, Joseph (2013). A number sense intervention for low-income kindergartners at risk for mathematics difficulties. Journal of Learning Disabilities, 46(2), 166-181. https://doi.org/10.1177/0022219411410233

FERNÁNDEZ, Martha (2019). Rehabilitación neuropsicológica en niños con TDAH: ¿Qué dice la evidencia sobre el entrenamiento neurocognitivo? Revista Guillermo de Ockham, 17(1), 65-76. https://doi.org/10.21500/22563202.3958 
FRAZIER, Thomas W., YOUNGSTROM, Eric. A., GLUTTING, Joseph J., \& WATKINS, Marley W. (2007). ADHD and achievement: Meta-analysis of the child, adolescent, and adult literatures and a concomitant study with college students. Journal of Learning Disabilities, 4O(1), 49-65. https://doi.org/10.1177/00222194070400010401

FRIEDMAN, Lauren M., RAPPORT, Mark D., ORBAN, Sarah A., ECKRICH, Samuel J., \& CALUB, Catrina A. (2018). Applied problem solving in children with ADHD: The mediating roles of working memory and mathematical calculation. Journal of Abnormal Child Psychology, 46(3), 491-504. https://doi.org/10.1007/s10802-017-0312-7

GEARY, David C. (2011). Cognitive predictors of achievement growth in mathematics: a 5-year longitudinal study. Developmental Psychology, 47(6), 1539. https://doi.org.10.1037/ a0025510

GEARY, David C., HOARD, Mary K., BYRD-CRAVEN, Jennifer, \& DESOTO, M. Catherine (2004). Strategy choices in simple and complex addition: Contributions of working memory and counting knowledge for children with mathematical disability. Journal of Experimental Child Psychology, 88(2), 121-151. https://doi.org/10.1016/j.jecp.2004.03.002

GREVEN, Corina U., KOVAS, Yulia, WILLCUTT, Erik G., PETRILL, Stephen A., \& PLOMIN, Robert (2014). Evidence for shared genetic risk between ADHD symptoms and reduced mathematics ability: a twin study. Journal of Child Psychology and Psychiatry, 55(1), 3948. https://doi.org/10.1111/jcpp.12090

KAUFMANN, Liane, \& NUERK, Hans C. (2008). Basic number processing deficits in ADHD: a broad examination of elementary and complex number processing skills in 9-to 12-year-old children with ADHD-C. Developmental Science, 11(5), 692-699. https://doi.org/10.1111/ j.1467-7687.2008.00718.x

KOFLER, Michael J., RAPPORT, Mark D., SARVER, Dustin E., RAIKER, Joseph S., ORBAN, Sarah A., FRIEDMAN, Lauren M., \& KOLOMEYER, Ellen G. (2013). Reaction time variability in ADHD: a meta-analytic review of 319 studies. Clinical Psychology Review, 33(6), 795-811. https://doi.org/10.1016/j.cpr.2013.06.001

MARZOCCHI, Gian. M., LUCANGELI, Daniela, DE MEO, Tiziana, FINI, Federica, \& CORNOLDI, Cesare (2002). The disturbing effect of irrelevant information on arithmetic problem solving in inattentive children. Developmental Neuropsychology, 21(1), 73-92. https://doi. org/10.1207/S15326942DN2101_4

MIRANDA-CASAS, Ana, MELIÁ-DE ALBA, Amanda, MARCO-TAVERNER, Rafaela, ROSELLÓ, Belén, \& MULAS, Fernando (2006). Dificultades en el aprendizaje de matemáticas en niños con trastorno por déficit de atención e hiperactividad. Revista de Neurología, 42(2), 163-170. DOI: https://doi.org/10.33588/rn.42S02.2005788 
MOLINAR, Judith, \& CERVANTES, Ana (2021). Actitudes percibidas en el discurso narrativo docente hacia el alumnado con TDAH. Un estudio de observación indirecta. Revista de Estudios y Experiencias en Educación, 20(42), 87-100. https://doi.org/10.21703/rexe. 20212042 molinar5

ORBACH, Lars.; HERZOG, Moritz; \& FRITZ, Anne (2020). Relation of attention deficit hyperactivity disorder (ADHD) to basic number skills and arithmetic fact retrieval in children. Research in Developmental Disabilities, 103, 103697; https://doi.org/10.1016/j.ridd.2020.103697

PASSOLUNGHI, María. C., \& SIEGEL, Linda. S. (2004). Working memory and access to numerical information in children with disability in mathematics. Journal of Experimental Child Psychology, 88(4), 348-367. https://doi.org/10.1016/j.jecp.2004.04.002

PASSOLUNGHI, María C., MARZOCCHI, Gian M., \& Fiorillo, Francesca (2005). Selective effect of inhibition of literal or numerical irrelevant information in children with attention deficit hyperactivity disorder (ADHD) or arithmetic learning disorder (ALD). Developmental Neuropsychology, 28(3), 731-753. https://doi.org/10.1207/s15326942dn2803_1

PENNINGTON, Bruce. F., \& OZONOFF, Sally (1996). Executive functions and developmental psychopathology. Journal of Child Psychology and Psychiatry, 37(1), 51-87. https://doi. org/10.1111/j.1469-7610.1996.tb01380.x

POLANCZYK, Gilherme V., WILLCUTT, Erik G., SALUM, Giovanni A., KIELING, Christian, \& ROHDE, Luise A. (2014). ADHD prevalence estimates across three decades: an updated systematic review and meta-regression analysis. International Journal of Epidemiology, 43(2), 434442. https://doi.org/10.1093/ije/dyt261

RUBINSTEN, Onri, \& HENIK, Avishai (2009). Developmental dyscalculia: Heterogeneity might not mean different mechanisms. Trends in Cognitive Sciences, 13(2), 92-99 https://doi.org/ 10.1016/j.tics.2008.11.002

VIEIRO, Pilar, PEREIRA, Ricardo \&, ROSENDE, Marta (2011). Gesmedición: Un programa on-line para la evaluación del proceso lector. En: Barca-Lozano, Alfonso \& Brenlla-Blanco, Juan Carlos (Eds.), Actas del XI Congreso Internacional Galego-Portugués de Psicopedagoxia.

Fecha de recepción: 28 de abril de 2021.

Fecha de revisión: 14 de junio de 2021.

Fecha de aceptación: 15 de junio de 2021.

Fecha de publicación: 1 de julio de 2021. 